\title{
A QUADRATURE FORMULA FOR ENTIRE FUNCTIONS OF EXPONENTIAL TYPE
}

\author{
QAZI IBADUR RAHMAN AND GERHARD SCHMEISSER
}

\begin{abstract}
A quadrature formula over a semi-infinite interval for entire functions of exponential type is established. An alternative approach to a known expansion formula and an extension of it are also presented.
\end{abstract}

\section{INTRODUCTION AND STATEMENT OF RESULTS}

It is known ([4]; see [2] and [12, (2.6)] for references) that if $f$ is an entire function of exponential type less than $2 \pi$ and $f$ is integrable in the sense of Cauchy on $\mathbf{R}$, then

$$
\int_{-\infty}^{\infty} f(x) d x=\sum_{n=-\infty}^{\infty} f(n)
$$

The restriction imposed on the growth of $f$ cannot be relaxed. Indeed, for the function

$$
f(z):= \begin{cases}\frac{\sin (2 \pi z)}{z} & \text { if } z \neq 0, \\ 2 \pi & \text { if } z=0,\end{cases}
$$

which is of order 1 type $2 \pi$, we have $\int_{-\infty}^{\infty} f(x) d x=\pi$ while $\sum_{-\infty}^{\infty} f(n)=2 \pi$. It is natural to wonder if a similarly simple formula holds for the integral over a half-line. Since for even functions, (1) is equivalent to

$$
\int_{0}^{\infty} f(x) d x=\frac{1}{2} f(0)+\sum_{n=1}^{\infty} f(n),
$$

it is meaningful to compare $\int_{0}^{\infty} f(x) d x$ with $\frac{1}{2} f(0)+\sum_{n=1}^{\infty} f(n)$. According to a formula of Plana $[8 ; 6, \S 4.9]$,

$$
\int_{0}^{\infty} f(x) d x=\frac{1}{2} f(0)+\sum_{n=1}^{\infty} f(n)-i \int_{0}^{\infty} \frac{f(i y)-f(-i y)}{e^{2 \pi y}-1} d y
$$

if

(a) $\lim _{y \rightarrow+\infty}|f(x \pm i y)| e^{-2 \pi y}=0$ uniformly in $x$ on every finite interval and

Received by the editor June 8, 1993.

1991 Mathematics Subject Classification. Primary 65D30, 65D32; Secondary 30D99. 
(b) $\int_{0}^{\infty}|f(x \pm i y)| e^{-2 \pi y} d y$ exists for every $x \geq 0$ and tends to zero as $x \rightarrow+\infty$. However, (2) expresses the quantity

$$
Q:=\int_{0}^{\infty} f(x) d x-\left(\frac{1}{2} f(0)+\sum_{n=1}^{\infty} f(n)\right)
$$

as an integral involving values of $f$ on the imaginary axis. Below we obtain an expression for $Q$ which is quite different and seems to us to be preferable from a numerical point of view.

Theorem 1. If $f$ is an entire function of exponential type $\tau$ less than $2 \pi$ and $\int_{0}^{\infty} f(x) d x$ exists in the sense of Cauchy, then

$$
\int_{0}^{\infty} f(x) d x=\frac{1}{2} f(0)+\sum_{n=1}^{\infty} f(n)+\sum_{j=1}^{\infty} f^{(2 j-1)}(0) \frac{B_{2 j}}{(2 j) !},
$$

where $B_{\nu}(\nu \in \mathbf{N})$ are the Bernoulli numbers. The second series converges absolutely.

Note that (1) follows from Theorem 1 applied to $f(x)+f(-x)$. For another formula for quadrature over semi-infinite intervals see [11, formula (1.13)].

The second series in (3) may not be small and so should not be seen as a remainder. However, we may have to include only the first few terms of this series to obtain a good approximation to the integral. This is indicated by

Theorem 2. If, in addition to the hypothesis of Theorem 1, $f$ is bounded by $M$ on the real line, then for $k \geq 2$

$$
\int_{0}^{\infty} f(x) d x=\frac{1}{2} f(0)+\sum_{n=1}^{\infty} f(n)+\sum_{j=1}^{k-1} f^{(2 j-1)}(0) \frac{B_{2 j}}{(2 j) !}+R_{k}[f]
$$

with

$$
\left|R_{k}[f]\right| \leq \frac{2 M \zeta(2 k)}{\left(1-(\tau / 2 \pi)^{2}\right) \tau}\left(\frac{\tau}{2 \pi}\right)^{2 k},
$$

where $\zeta(x):=\sum_{n=1}^{\infty} n^{-x}$ denotes the Riemann zeta function.

Remark 1. If $f$ is an entire function of exponential type $\tau$, then $f(h \cdot)$ is an entire function of exponential type less than $2 \pi$ for all $h \in(0,2 \pi / \tau)$. Since

$$
\int_{0}^{\infty} f(x) d x=h \int_{0}^{\infty} f(h x) d x
$$

we see that the restriction on the exponential type in Theorems 1 and 2 does not really limit their scope. Choosing $h$ appropriately and working with $f(h \cdot)$ instead of $f$ makes the right-hand side of (4) small in any case.

Remark 2. The assumption of boundedness of $f$ on the real line can be relaxed. As the proof of Theorem 2 will show, (4) holds with $\tau$ replaced by $\sigma$ if in addition to the hypothesis of Theorem 1 there exist constants $M>0$ and $\sigma \in(0,2 \pi)$ such that $\left|f^{(j)}(0)\right| \leq M \sigma^{j}$ for $j=3,5,7, \ldots$. 
Remark 3. A bound for the remainder $R_{k}[f]$ can be given even if $f$ satisfies nothing but the hypothesis of Theorem 1 . Indeed, for given $\varepsilon \in(0,2 \pi-\tau)$ there exists a $k_{0} \in \mathbf{N}$ such that

$$
\left|R_{k}[f]\right| \leq \frac{2 \zeta(2 k)}{2 \pi-\varepsilon} \cdot \frac{4 \pi^{2}}{\varepsilon(4 \pi-\varepsilon)}\left(1-\frac{\varepsilon}{2 \pi}\right)^{2 k} \quad \text { for } k \geq k_{0}
$$

This will be explained in $\S 3$.

For functions which are bounded on the real line, formula (3) can be extended as follows.

Theorem 3. Under the hypothesis of Theorem 2,

$$
\int_{0}^{\infty} f(x) d x=\sum_{n=0}^{\infty} f(z+n)+\sum_{j=1}^{\infty} f^{(j-1)}(0) \frac{B_{j}(z)}{j !},
$$

where $B_{j}(z) \quad(j \in \mathbf{N})$ denote the Bernoulli polynomials. Both series converge uniformly on compact subsets of $\mathbf{C}$. Moreover, the second series converges absolutely.

Remark 4. In Theorems $1-3$ the series over $n$ need not converge absolutely. For this, it suffices to consider the function

$$
f(z):= \begin{cases}\frac{\cos (\pi z)}{z+1 / 2} & \text { for } z \neq-1 / 2 \\ \pi & \text { for } z=-1 / 2\end{cases}
$$

which satisfies the hypotheses of these theorems.

Remark 5. As the proof will show, the conclusion of Theorem 3 remains true under the weaker hypothesis that in addition to the assumptions of Theorem 1 there exist constants $M>0$ and $\sigma \in(0,2 \pi)$ such that $\sup _{x \geq 0}\left|f^{(j)}(x)\right| \leq M \sigma^{j}$ for all $j \in \mathbf{N}$.

\section{LEMMAS}

We continue to use the notation $B_{j}$ and $\zeta(\cdot)$ for the $j$ th Bernoulli number and the Riemann zeta function, respectively. For easy reference, we formulate a few lemmas, which are not all new. The following lemma is the special case $m=0, a=0, b=N$ of Theorem 7 in [9].

Lemma 1. Let $f$ be holomorphic and of exponential type less than $2 \pi$ in a strip $\{z \in \mathbf{C}:-\delta \leq \operatorname{Re} z \leq N+\delta\}$, where $\delta>0$ and $N \in \mathbf{N}$. Then

$$
\begin{aligned}
\int_{0}^{N} f(x) d x= & \frac{1}{2}(f(0)+f(N))+\sum_{n=1}^{N-1} f(n) \\
& -\sum_{j=1}^{k}\left(f^{(2 j-1)}(N)-f^{(2 j-1)}(0)\right) \frac{B_{2 j}}{(2 j) !} \\
& +i(-1)^{k} \int_{0}^{\infty} L_{2 k}(t)\left(f^{(2 k)}(N+i t)-f^{(2 k)}(N-i t)\right. \\
& \left.-f^{(2 k)}(i t)+f^{(2 k)}(-i t)\right) d t
\end{aligned}
$$


with

$$
L_{k}(z):=(-1)^{k} \sum_{\nu=1}^{\infty} \frac{e^{-2 \pi \nu z}}{(2 \pi \nu)^{k}} \quad(k \in \mathbf{N} \backslash\{1\}, \operatorname{Re} z \geq 0) .
$$

The estimate contained in the following lemma will be used repeatedly.

Lemma 2. Let $f$ be an entire function of exponential type $\tau$. Then for given $z_{0} \in \mathbf{C}$ and $\varepsilon>0$, there exists an $n_{0} \in \mathbf{N}$ such that $\left|f^{(n)}\left(z_{0}\right)\right| \leq(\tau+\varepsilon)^{n}$ for $n \geq n_{0}$.

Proof. This follows from (2.2.11) in [1], taking into account the definition of exponential type as given in $[1, \S 2.1]$.

The following two lemmas are of importance for the investigation of the cases $N \rightarrow \infty$ and $k \rightarrow \infty$, respectively, in formula (8).

Lemma 3. If $f$ is an entire function of exponential type less than $2 \pi$ and $\int_{0}^{\infty} f(x) d x$ exists in the sense of Cauchy, then $f(x)$ and all the derivatives $f^{(j)}(x)$ tend to zero as $x \rightarrow+\infty$. Furthermore,

$$
\lim _{N \rightarrow \infty} \int_{0}^{\infty} L_{k}(t) f^{(k)}(N \pm i t) d t=0 .
$$

Proof. Denoting the exponential type of $f$ by $\tau$, we first observe $[1, \S 2.4]$ that along with $f$ all its derivatives are also of exponential type $\tau$. Further [1, Theorem 11.3.4*], the existence of $\int_{0}^{\infty} f(x) d x$ implies that $f(x)$ and all its derivatives $f^{(k)}(x)$ tend to zero as $x \rightarrow+\infty$. In particular, $f^{(k)}(x)$ is bounded on $[0,+\infty)$. Using all this information, we infer from Theorem 6.2.3 in [1] that

$$
\left|f^{(k)}(x+i y)\right| \leq C e^{(\pi+\tau / 2)|y|} \text { for } x \in[0,+\infty), y \in \mathbf{R}
$$

with an appropriate constant $C>0$. Thus, recalling (9), we have for $y>0$

$$
\begin{aligned}
& \left|\int_{y}^{\infty} L_{k}(t) f^{(k)}(N \pm i t) d t\right| \leq C\left|\int_{y}^{\infty} \sum_{\nu=1}^{\infty} \frac{e^{-2 \pi \nu t+\pi t+\tau t / 2}}{(2 \pi \nu)^{k}} d t\right| \\
& \quad \leq \frac{C}{(2 \pi)^{k}} \zeta(k) \int_{y}^{\infty} e^{-(\pi-\tau / 2) t} d t=\frac{C}{(2 \pi)^{k}} \frac{\zeta(k)}{\pi-\tau / 2} e^{-(\pi-\tau / 2) y},
\end{aligned}
$$

which tends to zero as $y \rightarrow+\infty$. On the other hand, the above observations imply [1, Theorem 6.2.8] that $f^{(k)}(x+i y)$ tends to zero as $x \rightarrow+\infty$ uniformly for $|y| \leq y_{0}$, where $y_{0}$ is any positive number. Hence,

$$
\lim _{N \rightarrow \infty} \int_{0}^{y} L_{k}(t) f^{(k)}(N \pm i t) d t=0
$$

for any positive $y$. This together with (11) implies (10).

Lemma 4. Let $f$ be an entire function of exponential type less than $2 \pi$. Then

$$
\lim _{k \rightarrow \infty} \int_{0}^{\infty} L_{k}(t) f^{(k)}(t) d t=0 .
$$

Proof. First we note that the integral $I_{k}:=\int_{0}^{\infty} L_{k}(t) f^{(k)}(t) d t$ exists for all $k \geq 2$, since along with $f$ the function $f^{(k)}$ is also of exponential type less than $2 \pi$. In view of the fact that $L_{k}(t)=L_{k+1}^{\prime}(t)$, integration by parts yields

$$
I_{k}=\left[L_{k+1}(t) f^{(k)}(t)\right]_{0}^{\infty}-\int_{0}^{\infty} L_{k+1}(t) f^{(k+1)}(t) d t=-L_{k+1}(0) f^{(k)}(0)-I_{k+1},
$$


and so for any $k>2$

$$
I_{2}=\sum_{j=2}^{k-1}(-1)^{j+1} L_{j+1}(0) f^{(j)}(0)+(-1)^{k} I_{k}
$$

It is therefore sufficient to show that

$$
I_{2}=\sum_{j=2}^{\infty}(-1)^{j+1} L_{j+1}(0) f^{(j)}(0)
$$

For this, we note that $I_{2}=\lim _{T \rightarrow \infty} \int_{0}^{T} L_{2}(t) f^{\prime \prime}(t) d t$ and write $f^{\prime \prime}$ as

$$
f^{\prime \prime}(t)=\sum_{n=0}^{\infty} \frac{f^{(n+2)}(0)}{n !} t^{n}
$$

where the series converges uniformly on compact subsets of $\mathbf{C}$. It follows that

$$
\int_{0}^{T} L_{2}(t) f^{\prime \prime}(t) d t=\sum_{n=0}^{\infty} \frac{f^{(n+2)}(0)}{n !} \int_{0}^{T} L_{2}(t) t^{n} d t \quad(T>0) .
$$

Integrating by parts, we get

$$
\begin{aligned}
\int_{0}^{\infty} L_{2}(t) t^{n} d t & =-n \int_{0}^{\infty} L_{3}(t) t^{n-1} d t=n(n-1) \int_{0}^{\infty} L_{4}(t) t^{n-2} d t \\
& =\cdots=(-1)^{n+1} n ! L_{n+3}(0) .
\end{aligned}
$$

Further,

$$
(-1)^{n+1} L_{n+3}(0)=\sum_{j=1}^{\infty} \frac{1}{(2 \pi j)^{n+3}}=\frac{1}{(2 \pi)^{n+3}} \zeta(n+3) \leq \frac{1}{(2 \pi)^{n+3}} \zeta(3) .
$$

Since $L_{2}(t)>0$, the estimates $(14)-(15)$ imply

$$
\left|\frac{f^{(n+2)}(0)}{n !} \int_{0}^{T} L_{2}(t) t^{n} d t\right| \leq\left|f^{(n+2)}(0) L_{n+3}(0)\right| \leq \frac{\left|f^{(n+2)}(0)\right|}{(2 \pi)^{n+2}} \frac{\zeta(3)}{2 \pi}
$$

for all $T>0$. By Lemma 2, the right-hand side of (10) converges geometrically to zero as $n \rightarrow \infty$. Hence, the series in (13) has an absolutely convergent majorant independent of $T$. Letting $T$ tend to infinity and taking into account (14), we thus obtain

$$
I_{2}=\int_{0}^{\infty} L_{2}(t) f^{\prime \prime}(t) d t=\sum_{n=0}^{\infty}(-1)^{n+1} L_{n+3}(0) f^{(n+2)}(0) .
$$

In view of (12) this implies that $\lim _{k \rightarrow \infty} I_{k}=0$ as was to be shown.

We also need an estimate for Bernoulli polynomials, which is so straightforward that it may not be necessary to look for a possible origin.

Lemma 5. The Bernoulli polynomials $B_{n}(z)$ satisfy

$$
\left|\frac{B_{n}(z)}{n !}\right| \leq \sum_{\nu=0}^{n}\left|\frac{B_{\nu} z^{n-\nu}}{\nu !(n-\nu) !}\right| \leq \frac{\pi^{2}}{3} \frac{e^{2 \pi|z|}}{(2 \pi)^{n}}
$$


Proof. The first inequality is obvious, since the Bernoulli polynomials may be expressed in terms of the Bernoulli numbers as $[7, \mathrm{p}$. 458, problem $4 ; 10, \mathrm{p}$. 224]

$$
B_{n}(z)=\sum_{\nu=0}^{n}\left(\begin{array}{l}
n \\
\nu
\end{array}\right) B_{\nu} z^{n-\nu} .
$$

Recalling that $[6$, p. 13 , p. 111 , p. 269 , eq. $(4.9-5) ; 10, \S 9.1]$

$$
\begin{gathered}
B_{0}=1, \quad B_{1}=-\frac{1}{2}, \\
B_{2 j}=\frac{(-1)^{j+1} 2(2 j) !}{(2 \pi)^{2 j}} \zeta(2 j), \quad B_{2 j+1}=0 \quad(j \in \mathbf{N}),
\end{gathered}
$$

we may write

$$
\sum_{\nu=0}^{n}\left|\left(\begin{array}{l}
n \\
\nu
\end{array}\right) B_{\nu} z^{n-\nu}\right| \leq|z|^{n}+\frac{n}{2}|z|^{n-1}+2 \sum_{\nu=2}^{n} \frac{\zeta(\nu)}{(2 \pi)^{\nu}} \frac{n !}{(n-\nu) !}|z|^{n-\nu} .
$$

Since $\zeta(\nu) \geq \zeta(2)=\pi^{2} / 6$ for $\nu \geq 2$, we get

$$
\begin{aligned}
\sum_{\nu=0}^{n}\left|\frac{B_{\nu} z^{n-\nu}}{\nu !(n-\nu) !}\right| & \leq \frac{|z|^{n}}{n !}+\frac{1}{2} \frac{|z|^{n-1}}{(n-1) !}+\frac{2 \zeta(2)}{(2 \pi)^{n}} \sum_{\nu=0}^{n-2} \frac{|2 \pi z|^{\nu}}{\nu !} \\
& \leq \frac{2 \zeta(2)}{(2 \pi)^{n}}\left(\frac{3}{\pi^{2}} \frac{|2 \pi z|^{n}}{n !}+\frac{3}{\pi} \frac{|2 \pi z|^{n-1}}{(n-1) !}+\sum_{\nu=0}^{n-2} \frac{|2 \pi z|^{\nu}}{\nu !}\right) \\
& \leq \frac{\pi^{2}}{3} \frac{1}{(2 \pi)^{n}} \sum_{\nu=0}^{n} \frac{|2 \pi z|^{\nu}}{\nu !},
\end{aligned}
$$

which readily confirms the second inequality in (17).

The following lemma is not new (see [3, formula (9.5)], where it has been deduced from a theorem based on the Borel transform and involving intricate arguments), but we shall present an alternative proof.

Lemma 6. If $f$ is an entire function of exponential type less than $2 \pi$, then

$$
f(z)=\int_{0}^{1} f(x) d x+\sum_{n=1}^{\infty}\left(f^{(n-1)}(1)-f^{(n-1)}(0)\right) \frac{B_{n}(z)}{n !} .
$$

Proof. Setting $N=1$ in Lemma 1 and letting $k$ tend to infinity, we infer from (8) with the help of Lemma 4 that

$$
\int_{0}^{1} f(x) d x=\frac{1}{2}(f(0)+f(1))-\sum_{j=1}^{\infty}\left(f^{(2 j-1)}(1)-f^{(2 j-1)}(0)\right) \frac{B_{2 j}}{(2 j) !} .
$$

Using that $B_{1}=-1 / 2$ and $B_{2 j+1}=0$ for $j \in \mathbf{N}$, we may rewrite (21) as

$$
f(0)=\int_{0}^{1} f(x) d x+\sum_{n=1}^{\infty}\left(f^{(n-1)}(1)-f^{(n-1)}(0)\right) \frac{B_{n}}{n !} .
$$


This formula applies to $f^{(k)}$ as well, since along with $f$ its derivatives also satisfy the hypothesis of Lemma 6 . Hence,

$$
\begin{aligned}
f^{(k)}(0) & =f^{(k-1)}(1)-f^{(k-1)}(0)+\sum_{n=1}^{\infty}\left(f^{(k+n-1)}(1)-f^{(k+n-1)}(0)\right) \frac{B_{n}}{n !} \\
& =\sum_{n=k}^{\infty}\left(f^{(n-1)}(1)-f^{(n-1)}(0)\right) \frac{B_{n-k}}{(n-k) !} .
\end{aligned}
$$

Substituting (22)-(23) into the partial sums of the Taylor series of $f$ at the origin, we get

$$
\begin{aligned}
\sum_{k=0}^{N} \frac{f^{(k)}(0)}{k !} z^{k}= & \int_{0}^{1} f(x) d x+\sum_{n=1}^{N}\left(f^{(n-1)}(1)-f^{(n-1)}(0)\right) \sum_{k=0}^{n} \frac{B_{n-k}}{(n-k) !} \frac{z^{k}}{k !} \\
& +\sum_{n=N+1}^{\infty}\left(f^{(n-1)}(1)-f^{(n-1)}(0)\right) \sum_{k=0}^{N} \frac{B_{n-k}}{(n-k) !} \frac{z^{k}}{k !}
\end{aligned}
$$

Now, by (18),

$$
\sum_{k=0}^{n} \frac{B_{n-k}}{(n-k) !} \frac{z^{k}}{k !}=\frac{B_{n}(z)}{n !}
$$

and by Lemma 5 for $n \geq N$,

$$
\left|\sum_{k=0}^{N} \frac{B_{n-k}}{(n-k) !} \frac{z^{k}}{k !}\right| \leq \frac{\pi^{2}}{3} \frac{e^{2 \pi|z|}}{(2 \pi)^{n}} .
$$

Hence, denoting by $\tau \in(0,2 \pi)$ the exponential type of $f$ and using Lemma 2 with $\varepsilon=\pi-\tau / 2$, we find that for sufficiently large $N$

$$
\begin{aligned}
& \left|\sum_{n=N+1}^{\infty}\left(f^{(n-1)}(1)-f^{(n-1)}(0)\right) \sum_{k=0}^{N} \frac{B_{n-k}}{(n-k) !} \frac{z^{k}}{k !}\right| \\
& \quad \leq \frac{2 \pi^{2}}{3} \frac{e^{2 \pi|z|}}{\pi+\tau / 2} \sum_{n=N+1}^{\infty}\left(\frac{2 \pi+\tau}{4 \pi}\right)^{n}=\frac{16 \pi^{3}}{3} \frac{e^{2 \pi|z|}}{4 \pi^{2}-\tau^{2}}\left(\frac{2 \pi+\tau}{4 \pi}\right)^{N+1},
\end{aligned}
$$

which converges geometrically to zero as $N \rightarrow \infty$. This shows that (20) is the limit of (24) as $N$ tends to infinity.

It was the late Professor R. P. Boas who asked one of us whether the ideas developed in [9] could be used to establish formula (20) of Lemma 6.

\section{Proofs of the theorems}

Proof of Theorem 1. Letting $N$ tend to infinity in Lemma 1, we infer from (8) with the help of Lemma 3 that

$$
\begin{aligned}
\int_{0}^{\infty} f(x) d x= & \frac{1}{2} f(0)+\sum_{n=1}^{\infty} f(n)+\sum_{j=1}^{k} f^{(2 j-1)}(0) \frac{B_{2 j}}{(2 j) !} \\
& -i(-1)^{k} \int_{0}^{\infty} L_{2 k}(t)\left(f^{(2 k)}(i t)-f^{(2 k)}(-i t)\right) d t
\end{aligned}
$$


(see also [9, Corollary 6]). The convergence of the infinite series is obtained as a consequence. Since along with $f$ the functions $f( \pm i \cdot)$ are also of exponential type less than $2 \pi$, we see, using Lemma 4 , that the right-hand side of $(25)$ tends to that of (3) as $k \rightarrow \infty$, thereby entailing the convergence of the series with respect to $j$. The absolute convergence of this series follows readily by estimating its terms with the help of Lemma 2 and (19).

Proof of Theorem 2. According to Theorem 1,

$$
R_{k}[f]=\sum_{j=k}^{\infty} f^{(2 j-1)}(0) \frac{B_{2 j}}{(2 j) !} .
$$

Repeated application of Bernstein's inequality for entire functions of exponential type [1, Theorem 11.1.2] yields

$$
\left|f^{(j)}(0)\right| \leq M \tau^{j}
$$

Thus, (26) and (19) lead to

$$
\left|R_{k}[f]\right| \leq M \sum_{j=k}^{\infty} \frac{2 \zeta(2 j)}{(2 \pi)^{2 j}} \tau^{2 j-1} \leq \frac{2 M \zeta(2 k)}{\tau} \sum_{j=k}^{\infty}\left(\frac{\tau}{2 \pi}\right)^{2 j}
$$

which clearly shows that (4) holds.

Justification of Remarks 2-3. For the crucial estimate (27) to hold, the boundedness of $f$ on $\mathbf{R}$ is (by virtue of Bernstein's inequality) a sufficient, but not necessary, condition, as the example $f(z):=e^{-z}$ shows. Moreover, (27) is needed for odd $j$ only. This justifies Remark 2 .

Under the hypothesis of Theorem 1, we infer from Lemma 2 that $\left|f^{(j)}(0)\right| \leq$ $(2 \pi-\varepsilon)^{j}$ for $\varepsilon \in(0,2 \pi-\tau)$ and all sufficiently large $j$. Using this inequality instead of (27) in the proof of Theorem 2, we easily confirm (5), and so Remark 3 is justified.

Proof of Theorem 3. If we apply formula (20) of Lemma 6 to the function $f(\cdot+\nu)$, summation over $\nu$ yields

$$
\sum_{\nu=0}^{N} f(z+\nu)=\int_{0}^{N} f(x) d x+\sum_{n=1}^{\infty}\left(f^{(n-1)}(N)-f^{(n-1)}(0)\right) \frac{B_{n}(z)}{n !} .
$$

We now claim that

$$
S_{N}(z):=\sum_{n=1}^{\infty} f^{(n-1)}(N) \frac{B_{n}(z)}{n !}
$$

tends to zero as $N \rightarrow \infty$, the convergence being uniform with respect to $z$ on compact subsets of $\mathbf{C}$. Clearly, for fixed $n_{0} \geq 2$,

$$
\lim _{N \rightarrow \infty} \sum_{n=1}^{n_{0}-1} f^{(n-1)}(N) \frac{B_{n}(z)}{n !}=0,
$$

since by Lemma $3, f^{(j)}(N)(j=0,1, \ldots)$ all tend to zero. Further, by Bernstein's inequality for entire functions of exponential type [1, Theorem 11.1.2],

$$
\left|f^{(j)}(\xi)\right| \leq M \tau^{j} \quad \text { for any } \xi \in \mathbf{R} .
$$


Hence, using Lemma 5, we may estimate

$$
\begin{aligned}
\sum_{n=n_{0}}^{\infty}\left|f^{(n-1)}(\xi) \frac{B_{n}(z)}{n !}\right| & \leq \frac{\pi^{2}}{3} \frac{e^{2 \pi|z|}}{\tau} M \sum_{n=n_{0}}^{\infty}\left(\frac{\tau}{2 \pi}\right)^{n} \\
& =\frac{\pi^{2}}{3} \frac{e^{2 \pi|z|}}{\tau} \frac{M}{1-\tau /(2 \pi)}\left(\frac{\tau}{2 \pi}\right)^{n_{0}} .
\end{aligned}
$$

This together with (29) shows that $\lim _{N \rightarrow \infty} S_{N}(z)=0$, uniformly on compact subsets as claimed above. Thus, (6) follows from (28) by passing to the limit $N \rightarrow \infty$. Moreover, (31) implies that the second series on the right-hand side of $(6)$ converges absolutely and uniformly on compact subsets of C . Finally, applying (6) to $f(\cdot+N)$, we obtain

$$
\sum_{n=N}^{\infty} f(z+n)=\int_{N}^{\infty} f(x) d x-S_{N}(z)
$$

Therefore, the first series on the right-hand side of (6) converges also uniformly on compact subsets of $\mathbf{C}$.

Justification of Remark 5. In the proof of Theorem 3, the boundedness of $f$ on $\mathbf{R}$ was simply used to guarantee the validity of (30). However, this inequality is needed only for $\xi \geq 0$-a special case which does not necessarily require boundedness on the whole real line, as the example $f(z):=e^{-z}$ shows. This justifies Remark 5.

\section{NUMERICAL EXAMPLES}

For practical purposes it is reasonable to use the transformation in Remark 1 even when the hypothesis of Theorem 2 is satisfied. For an arbitrary $h \in$ $(0,2 \pi / \tau)$ the quadrature formula obtained in this way reads

$$
\int_{0}^{\infty} f(x) d x=\frac{h}{2} f(0)+h \sum_{n=1}^{\infty} f(h n)+\sum_{j=1}^{k-1} h^{2 j} f^{(2 j-1)}(0) \frac{B_{2 j}}{(2 j) !}+R_{k}(h)[f]
$$

where

$$
\left|R_{k}(h)[f]\right| \leq \frac{2 M \zeta(2 k)}{\left(1-(h \tau / 2 \pi)^{2}\right) \tau}\left(\frac{h \tau}{2 \pi}\right)^{2 k}
$$

Although the summation over $n$ is infinite, its terms are in general much simpler than those of the finite sum. Therefore, one should choose $h$ so small that $k$ can be kept relatively small. The summation over $j$ may then be handled by hand, while the summation over $n$ may be carried out on a computer.

Example 1. Let $f$ be the function defined in (7), which is not absolutely integrable and is therefore not admissible for most of the familiar quadrature schemes. Since $f$ is of exponential type $\pi$, Theorem 2 could be applied directly. However, we prefer (32), choosing $h=2^{-m} \quad\left(m \in \mathbf{N}_{0}\right)$, and obtain

$$
\begin{aligned}
\int_{0}^{\infty} f(x) d x= & 2^{-m}+\sum_{n=1}^{\infty} \frac{\cos \left(2^{-m} \pi n\right)}{n+2^{m-1}} \\
& +\sum_{j=1}^{k-1} 2^{-2 m j} f^{(2 j-1)}(0) \frac{B_{2 j}}{(2 j) !}+R_{k}\left(2^{-m}\right)[f] .
\end{aligned}
$$


It is known [5, p. 66, 1.441] that

$$
\sum_{n=1}^{\infty} \frac{\sin \left(2^{-m} \pi n\right)}{n}=\frac{\pi}{2}\left(1-2^{-m}\right)
$$

and so for $m \in \mathbf{N}$ we have

$$
\sum_{n=1}^{\infty} \frac{\cos \left(2^{-m} \pi n\right)}{n+2^{m-1}}=\sum_{n=2^{m-1}+1}^{\infty} \frac{\sin \left(2^{-m} \pi n\right)}{n}=\frac{\pi}{2}\left(1-2^{-m}\right)-\sum_{n=1}^{2^{m-1}} \frac{\sin \left(2^{-m} \pi n\right)}{n} .
$$

Further, for $m=0$ we have $[5$, p. 29, 0.232]

$$
\sum_{n=1}^{\infty} \frac{\cos \left(2^{-m} \pi n\right)}{n+2^{m-1}}=2 \sum_{n=1}^{\infty} \frac{(-1)^{n}}{2 n+1}=\frac{\pi}{2}-2=\frac{\pi}{2}\left(1-2^{-m}\right)-\left(2-\frac{\pi}{2}\right) .
$$

Hence, introducing

$$
Q_{m}:= \begin{cases}2-\frac{\pi}{2} & \text { if } m=0, \\ \sum_{n=1}^{2^{m-1}} \frac{\sin \left(2^{-m} \pi n\right)}{n} & \text { if } m \in \mathbf{N},\end{cases}
$$

we may rewrite (34) as

$$
\int_{0}^{\infty} f(x) d x=\frac{\pi}{2}\left(1-2^{-m}\right)+2^{-m}-Q_{m}+\sum_{j=1}^{k-1} 2^{-2 j m} f^{(2 j-1)}(0) \frac{B_{2 j}}{(2 j) !}+\Delta_{k, m},
$$

where

$$
\left|\Delta_{k, m}\right| \leq \frac{2 \zeta(2 k)}{1-4^{-(m+1)}} \frac{1}{4^{k(m+1)}} .
$$

In passing we mention that $(36)$ is equivalent to performing the calculations

$$
\begin{aligned}
\int_{0}^{\infty} f(x) d x & =\int_{0}^{\infty} \frac{\cos (\pi x)}{x+1 / 2} d x=\int_{\pi / 2}^{\infty} \frac{\sin x}{x} d x \\
& =\int_{0}^{\infty} \frac{\sin x}{x} d x-\int_{0}^{\pi / 2} \frac{\sin x}{x} d x=\frac{\pi}{2}-\int_{0}^{\pi / 2} \frac{\sin x}{x} d x
\end{aligned}
$$

and approximating the last integral by means of the Euler-Maclaurin formula over $[0, \pi / 2]$. Table 1 contains numerical values of the bound in (37). It is seen that $k=3$ already guarantees high accuracy. Denoting by $I_{k, m}:=$ $\int_{0}^{\infty} f(x) d x-\Delta_{k, m}$ the approximation to the integral, we have for $k=3$

$$
I_{3, m}=\frac{\pi}{2}-\left(\frac{\pi}{2}-1\right) 2^{-m}-\frac{1}{3} 2^{-2 m}-\frac{\pi^{2}-8}{60} 2^{-4 m}-Q_{m}
$$

with $Q_{m}$ defined in (35). Numerical results obtained with this formula are given in Table 2.

Example 2. Let $f(x):=e^{-x} \sin x$. Since for complex argument we may write

$$
f(z)=\frac{e^{(-1+i) z}-e^{-(1+i) z}}{2 i}
$$


TABLE 1. Numerical values of the bounds (37) for $\Delta_{k, m}$

\begin{tabular}{|c|ccccc|}
\hline$m \backslash k$ & 1 & 2 & 3 & 4 & 5 \\
\hline 0 & $1.10 \cdot 10^{+00}$ & $1.80 \cdot 10^{-01}$ & $4.24 \cdot 10^{-02}$ & $1.05 \cdot 10^{-02}$ & $2.61 \cdot 10^{-03}$ \\
1 & $2.19 \cdot 10^{-01}$ & $9.02 \cdot 10^{-03}$ & $5.30 \cdot 10^{-04}$ & $3.27 \cdot 10^{-05}$ & $2.04 \cdot 10^{-06}$ \\
2 & $5.22 \cdot 10^{-02}$ & $5.37 \cdot 10^{-04}$ & $7.88 \cdot 10^{-06}$ & $1.22 \cdot 10^{-07}$ & $1.89 \cdot 10^{-09}$ \\
3 & $1.29 \cdot 10^{-02}$ & $3.32 \cdot 10^{-05}$ & $1.22 \cdot 10^{-07}$ & $4.69 \cdot 10^{-10}$ & $1.83 \cdot 10^{-12}$ \\
4 & $3.22 \cdot 10^{-03}$ & $2.07 \cdot 10^{-06}$ & $1.90 \cdot 10^{-09}$ & $1.83 \cdot 10^{-12}$ & $1.78 \cdot 10^{-15}$ \\
5 & $8.03 \cdot 10^{-04}$ & $1.29 \cdot 10^{-07}$ & $2.96 \cdot 10^{-11}$ & $7.14 \cdot 10^{-15}$ & $1.74 \cdot 10^{-18}$ \\
6 & $2.01 \cdot 10^{-04}$ & $8.06 \cdot 10^{-09}$ & $4.63 \cdot 10^{-13}$ & $2.79 \cdot 10^{-17}$ & $1.70 \cdot 10^{-21}$ \\
7 & $5.02 \cdot 10^{-05}$ & $5.04 \cdot 10^{-10}$ & $7.23 \cdot 10^{-15}$ & $1.09 \cdot 10^{-19}$ & $1.66 \cdot 10^{-24}$ \\
8 & $1.25 \cdot 10^{-05}$ & $3.15 \cdot 10^{-11}$ & $1.13 \cdot 10^{-16}$ & $4.25 \cdot 10^{-22}$ & $1.62 \cdot 10^{-27}$ \\
\hline
\end{tabular}

TABLE 2. $I_{3, m}$ of Example 1

\begin{tabular}{|c|c|}
\hline$m$ & $I_{3, m}$ \\
\hline 0 & 0.2063029201100739 \\
1 & 0.2001173254796469 \\
2 & 0.2000354115397625 \\
3 & 0.2000341780436255 \\
4 & 0.2000341589429144 \\
5 & 0.2000341586451322 \\
6 & 0.2000341586404818 \\
7 & 0.2000341586404090 \\
8 & 0.2000341586404079 \\
\hline
\end{tabular}

we see that $|f(z)| \leq e^{\sqrt{2}|z|}$, and hence $f$ is of exponential type $\sqrt{2}$. The function $f$ is not bounded on $\mathbf{R}$, but from (38) a straightforward calculation yields

$$
f^{(2 j-1)}(0)=(-1)^{[(j-1) / 2]} 2^{j-1} \text { for } j \in \mathbf{N},
$$

where we used the Gauss notation $[x]$ for the integer part of $x$. Therefore, Remark 2 applies with $M=1 / \sqrt{2}$ and $\sigma=\sqrt{2}$. Moreover, (32) makes sense for all $h \in(0, \sqrt{2} \pi)$. In particular, if $h=2^{-m} \pi \quad\left(m \in \mathbf{N}_{0}\right)$, the estimate (33) becomes

$$
\left|R_{k}\left(2^{-m} \pi\right)[f]\right| \leq \frac{\zeta(2 k)}{1-2^{-(2 m+1)}} 2^{-k(2 m+1)} .
$$

Note that $m=0$ is admissible, in which case the infinite series in (32) disappears completely; however, the remainder would converge to zero only like $2^{-k}$ requiring large $k$. On the other hand, if $m$ is large, $k$ can be kept relatively small, but the infinite series converges slowly. Numerical results are given in Tables 3 and 4 (next page), where $I_{k, m}:=\int_{0}^{\infty} f(x) d x-R_{k}\left(2^{-m} \pi\right)[f]$ is again the approximation to the integral and $N$ denotes the number of evaluations of $f$ needed to calculate the infinite series with an error less than $5 \cdot 10^{-16}$. Note that the precise value of the integral is $1 / 2$, a primitive of $f$ being $-\frac{1}{2} e^{-x}(\cos x+\sin x)$.

Let us compare (32) with formula (1.13) in [11], which reads as follows

$$
\int_{0}^{\infty} f(x) d x=\log q \sum_{n=-\infty}^{\infty} q^{n} f\left(q^{n}\right)+R(q)[f] \quad(q>1) .
$$

For Example 1, this formula is not applicable, since the infinite series diverges. 
TABLE 3. Approximations $I_{k, m}$ of Example 2 for $k=1, \ldots, 4$

\begin{tabular}{|c|cccc|r|}
\hline$m \backslash k$ & 1 & 2 & 3 & 4 & $N$ \\
\hline 0 & 0.000000000000000 & 0.822467033424113 & 0.551886224996329 & 0.424718342248273 & 11 \\
1 & 0.313010082813037 & 0.518626841169065 & 0.501715540642329 & 0.499728542474391 & 22 \\
2 & 0.449682827105259 & 0.501087016694267 & 0.500030060411346 & 0.499999013564971 & 45 \\
3 & 0.487215493708023 & 0.500066541105274 & 0.500000481337592 & 0.4999999996230617 & 90 \\
4 & 0.496791374451324 & 0.500004136300637 & 0.500000007565157 & 0.499999999985361 & 180 \\
5 & 0.499197067702016 & 0.500000258164345 & 0.500000000118377 & 0.4999999999999943 & 359 \\
\hline
\end{tabular}

TABLE 4. Approximations $I_{k, m}$ of Example 2 for $k=5, \ldots, 8$

\begin{tabular}{|c|cccc|r|}
\hline$m \backslash k$ & 5 & 6 & 7 & 8 & $N$ \\
\hline 0 & 0.487473177010644 & 0.518754257483388 & 0.503125412380993 & 0.495312433879938 & 11 \\
1 & 0.499973678547681 & 0.500004226477830 & 0.500000410841819 & 0.499999933975455 & 22 \\
2 & 0.499999971127758 & 0.500000000959721 & 0.500000000028169 & 0.4999999999999063 & 45 \\
3 & 0.499999999971097 & 0.500000000000230 & 0.500000000000002 & 0.500000000000000 & 90 \\
4 & 0.499999999999972 & 0.500000000000000 & 0.500000000000000 & 0.500000000000000 & 179 \\
5 & 0.500000000000000 & 0.500000000000000 & 0.500000000000000 & 0.500000000000000 & 359 \\
\hline
\end{tabular}

TABLE 5. Approximations $I_{m}$ by (41)

\begin{tabular}{|c|c|c|r|}
\hline$m$ & $q_{m}$ & $I_{m}$ & $N$ \\
\hline 1 & 2.7182818 & 0.497592866827195 & 39 \\
2 & 1.6487213 & 0.499760107047253 & 79 \\
3 & 1.3956124 & 0.500002842086111 & 118 \\
4 & 1.2840254 & 0.500000001472323 & 158 \\
5 & 1.2214028 & 0.500000000003945 & 197 \\
6 & 1.1813604 & 0.500000000001427 & 237 \\
7 & 1.1535650 & 0.4999999999999996 & 277 \\
8 & 1.1331485 & 0.499999999999999 & 316 \\
\hline
\end{tabular}

For Example 2, (41) makes sense and according to Remark 1.5 in [11] we have for every $\beta \in(0,1 / 2)$

$$
R(q)[f]=\mathscr{O}\left(\exp \left(-\beta \pi^{2} / \log q\right)\right) \quad \text { as } q \rightarrow 1+.
$$

Hence, replacing $q$ by $q_{m}:=\exp (1 / m) \quad(m=1,2, \ldots)$, we see that the approximations to the integral $I_{m}:=\int_{0}^{\infty} f(x) d x-R\left(q_{m}\right)[f]$ converge at least geometrically and may, in view of (40), be compared with the previous approximations $I_{k, m}$. Numerical results are given in Table 5, where again $N$ denotes the number of evaluations of $f$.

It is seen from Tables 3-5 that as $m$ increases by 1 the number of correct digits obtained by the approximations $I_{k, m}$ and $I_{m}$ increases in the average by about $k / 2$ and 2 , respectively. Hence, formulae (41) and (32) are comparable if in the latter $k$ is chosen to be 4 . For $k>4$ formula (32) is superior (see Table 4).

Two further aspects should also be taken into account. Looking at the last columns of Tables 3-5, we see that if we want to restrict the number of evaluations of $f$ to about 100, then better approximations are obtained by formula (32) provided $k$ is at least 2; i.e., the second sum in (32) does not reduce to zero. In particular, in case $k=8$, already $N=90$ guarantees 15 correct digits, while formula (41) needs more than 277 evaluations of $f$ to achieve the same accuracy. However, in Tables $3-4, N$ roughly doubles as $m$ increases by 1 , 
whereas in Table $5, N$ always increases by about 40 . This means that for any fixed $k$, formula (32) is ultimately less efficient than (41) if the accuracy is forced up artificially.

It should be mentioned that formula (41) is very simple to implement, whereas formula (32) becomes more elaborate with increasing $k$. Indeed, the calculation of $f^{(2 j-1)}(0)$ requires preliminary work by hand, and the Bernoulli numbers have to be either prepared for input or generated by a subroutine.

\section{BIBLIOGRAPHY}

1. R. P. Boas, Jr., Entire functions, Academic Press, New York, 1954.

2. $\ldots$ Summation formulas and band-limited signals, Tôhoku Math. J. (2) 24 (1972), 121-125.

3. R. P. Boas, Jr. and R. C. Buck, Polynomial expansions and analytic functions, Springer, Berlin, 1958.

4. C. Frappier and Q. I. Rahman, Une formule de quadrature pour les fonctions entières de type exponentiel, Ann. Sci. Math. Québec 10 (1986), 17-26.

5. I. S. Gradstein and I. M. Ryshik, Tafeln-Tables, vol. 1 (bilingual: German-English), Harri Deutsch, Frankfurt/M., 1981.

6. P. Henrici, Applied and computational complex analysis, vol. 1, Wiley, New York, 1974.

7. __ Applied and computational complex analysis, vol. 2, Wiley, New York, 1977.

8. E. Lindelöf, Le calcul des résidus, Chelsea, New York, 1947.

9. Q. I. Rahman and G. Schmeisser, Quadrature formulae and functions of exponential type, Math. Comp. 54 (1990), 245-270.

10. G. Schmeisser and H. Schirmeier, Praktische Mathematik, de Gruyter, Berlin, 1976.

11. F. Stenger, Integration formulae based on the trapezoidal formula, J. Inst. Math. Appl. 12 (1973), 103-114.

12. _ Numerical methods based on Whittaker cardinal, or sinc functions, SIAM Rev. 23 (1981), 165-224.

Département de Mathématiques et de Statistique, Université de Montréal, Montréal, Québec, Canada H3C 3J7

$E$-mail address: rahmanqi@ere.umontreal.ca

Mathematisches Institut, Universität Erlangen-Nürnberg, Bismarckstrasse $1 \frac{1}{2}$, D-91054 ERLANGen, Germany

E-mail address: schmeis@mi.uni-erlangen.de 